\title{
An innovative energy management system acting on multiple battery packages
}

TBA

This manuskript is not available according to publishing restriction.

Thank you for your understanding.

(C) Springer Fachmedien Wiesbaden 2016, M. Bargende, H.-C. Reuss, J. Wiedemann (Hrsg.), 16. Internationales Stuttgarter Symposium, Proceedings, DOI 10.1007/978-3-658-13255-2_78 